\title{
Quantum and Raman Noise in a Depleted Fiber Optical Parametric Amplifier
}

Friis, Søren Michael Mørk; Rottwitt, Karsten; McKinstrie, Colin J.

Published in:

Nonlinear Optics Technical Digest

Publication date:

2013

Document Version

Publisher's PDF, also known as Version of record

Link back to DTU Orbit

Citation (APA):

Friis, S. M. M., Rottwitt, K., \& McKinstrie, C. J. (2013). Quantum and Raman Noise in a Depleted Fiber Optical Parametric Amplifier. In Nonlinear Optics Technical Digest (pp. NW4A.06). Optical Society of America.

\section{General rights}

Copyright and moral rights for the publications made accessible in the public portal are retained by the authors and/or other copyright owners and it is a condition of accessing publications that users recognise and abide by the legal requirements associated with these rights.

- Users may download and print one copy of any publication from the public portal for the purpose of private study or research.

- You may not further distribute the material or use it for any profit-making activity or commercial gain

- You may freely distribute the URL identifying the publication in the public portal

If you believe that this document breaches copyright please contact us providing details, and we will remove access to the work immediately and investigate your claim. 


\title{
Quantum and Raman Noise in a Depleted Fiber Optical Parametric Amplifier
}

\author{
Søren M. M. Friis ${ }^{1}$, Karsten Rottwitt ${ }^{1}$ and Colin J. McKinstrie ${ }^{2}$ \\ ${ }^{1}$ Department of Photonics Engineering, Technical University of Denmark, 2800 Kongens Lyngby, Denmark \\ ${ }^{2}$ Bell Laboratories, Alcatel Lucent, Holmdel, New Jersey, 07733, USA \\ smmf@fotonik.dtu.dk
}

\begin{abstract}
The noise properties of both phase-sensitive and phase-insensitive saturated parametric amplifiers are studied using a semi-classical approach. Vacuum fluctuations as well as spontaneous Raman scattering are included in the analysis.
\end{abstract}

(C) 2013 Optical Society of America

OCIS codes: (060.2320) Fiber optics amplifiers and oscillators, (270.2500) Fluctuations, relaxations, and noise.

\section{Summary}

The quantum noise properties of both phase-insensitive (PI) and phase-sensitive (PS) fiber optical parametric amplifiers (FOPAs) are well understood in the linear gain regime [1,2], and the effects of spontaneous Raman scattering has been described successfully [3-5]. However, previous work are carried out under the approximations of a constant pump power and has disregarded fiber loss. In this paper we do investigations of the noise properties of fiber optical parametric amplifiers that are valid in the linear as well as in the nonlinear gain regimes, and in which fiber attenuation, Raman scattering and quantum fluctuation are included.

The classical three-wave FOPA (signal, idler and degenerate pump) is modelled in a single-moded polarization maintaining $\chi^{(3)}$-nonlinear fiber according to ref. [6]. Fiber attenuation is included by adding a linear loss term to each wave component, and Raman scattering is modelled in the equation for wave $k$ with terms of $g_{R}\left(\Omega_{k l}\right)\left|A_{l}\right|^{2} A_{k} / 2$ to describe the interaction among $A_{k}$ and $A_{l}$ and a similar term for the interaction among $A_{k}$ and $A_{m}$ where $(k, l, m)=(p, s, i)$, corresponding to the pump, signal and idler waves. $\Omega_{k l}$ is the frequency shift between waves $k$ and $l$ and $g_{R}\left(\Omega_{k l}\right)$ is the Raman gain coefficient between the two waves. Spontaneous Raman scattering is described by including the thermal phonon equilibrium number $n_{T}\left(\Omega_{k l}\right)=\left(\exp \left(\hbar \Omega_{k l} / k_{B} T\right)-1\right)^{-1}$, where $\hbar$ and $k_{B}$ are Plancks and Boltzmanns constants and $T$ the temperature [7]. To obtain equations which are valid in the saturated pump regime, we do not neglect any interaction terms among the three waves; however, we neglect all higher order four wave mixing products, consequently the model is not valid in deep depletion.

Since the input fields must resemble coherent states, ensembles of classical fields with $2 \cdot 10^{4}$ elements are generated by dividing each field in their real and imaginary parts and then adding a normally distributed stochastic variable $\delta A$ with statistical properties $\langle\delta A\rangle=0$ and $\left\langle\delta A^{2}\right\rangle=\hbar \omega B_{0} / 4$ to each field, where $\omega$ is the frequency of the field and $B_{0}$ is the bandwidth of the field. This procedure simulates the quantum fluctuations in the two quadratures of the quantum electric field and thus correctly captures the uncertainty in field amplitude and phase.

Vacuum fluctuations are included by adding fluctuations to the fields during propagation. In each numerical step, $\Delta z$, the fiber attenuation is accounted for by adding $\delta a$ to each quadrature of the field with statistical properties $\langle\delta a\rangle=0$ and $\left\langle\delta a^{2}\right\rangle=\hbar \omega_{p} B_{0} \alpha \Delta z / 4$, where $\alpha$ is the linear loss coefficient; the expression originates from the quantum uncertainty relation of the variances of the two quadratures. Correspondingly, spontaneous Raman scattering from wave $l$ to $k$ is accounted for by adding yet another fluctuation with properties $\left\langle\delta a_{k l}\right\rangle=0$ and $\left\langle\delta a_{k l}^{2}\right\rangle=\left(1+n_{T}\left(\Omega_{k l}\right)\right) \hbar \omega_{l} B_{0} g_{R}\left(\Omega_{k l}\right)\left|A_{l}\right|^{2} \Delta z / 2$ to the equation for $k$, and a similar fluctuation is added for the contribution of wave $m$ to $k$.

We define an individual signal-to-noise ratio (SNR) for the PI amplifier to be $S N R_{P I}=\left\langle n_{S}\right\rangle^{2} / \operatorname{Var}\left(\mathrm{n}_{\mathrm{s}}\right)$ and a combined SNR for the PS amplifier to be $S N R_{P S}=\left\langle n_{s}+n_{i}\right\rangle^{2} / \operatorname{Var}\left(\mathrm{n}_{\mathrm{s}}+\mathrm{n}_{\mathrm{i}}\right)$, where $n_{s}$ and $n_{i}$ are the signal and idler photon numbers. The noise figure (NF) is defined for both cases as $N F=S N R_{\text {in }} / S N R_{\text {out }}$. Figure 1(a) shows the NF spectra of the PI and the PS amplifier with and without the Raman contribution. The initial phases of the input fields are $\phi_{p}=0$, $\phi_{s}=-\pi / 4$ and $\phi_{i}=\pi / 4$. In the PI case, spontaneous Raman scattering increases the NF asymmetrically compared to the $3 \mathrm{~dB}$ noise floor of the conventional FOPA without Raman; the local increase around the pump is caused by 


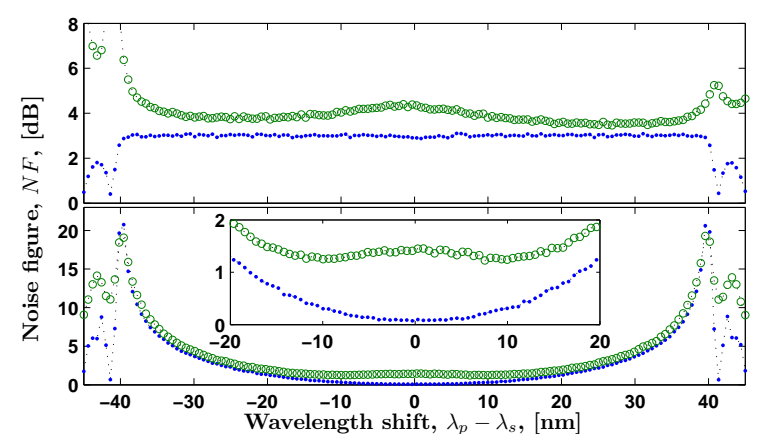

(a)

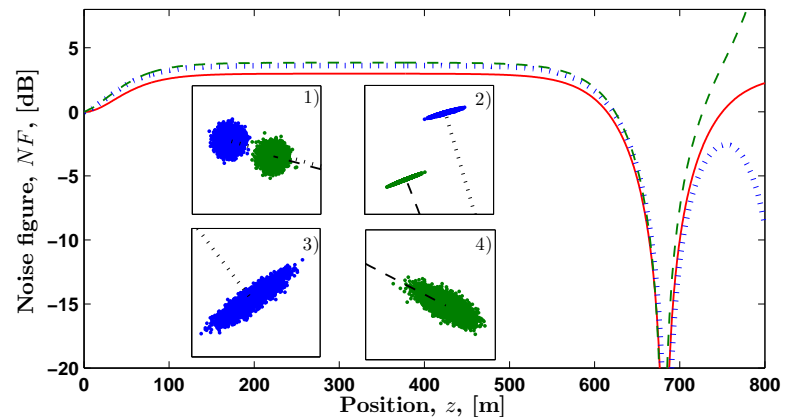

(b)

Fig. 1. (color online) (a) NF spectra of the PI (top) and PS (bottom) amplifiers w/o Raman (blue dots) and w/ Raman (green circles) for $300 \mathrm{~m}$ of fiber; inset shows a zoom. (b) NF vs. position in PI configuration for $\lambda_{s}=\lambda_{p}-28 \mathrm{~nm}$ (dashed green), $\lambda_{s}=\lambda_{p}+28 \mathrm{~nm}$ (dotted blue) and $\lambda_{s}=\lambda_{p}-28$ $\mathrm{nm}$ w/o Raman (solid red). Insets show constellation diagrams at 1) $z=300 \mathrm{~m}, 2$ ) $z=680 \mathrm{~m}$ and 3) and 4) $z=800 \mathrm{~m}$. Parameters from [6] are used with $\alpha=0.4 \mathrm{~dB} / \mathrm{km}, T=300 \mathrm{~K}$ and $\Delta z=1 \mathrm{~m}$.

the divergence in the phonon equilibrium number. In the PS case, Raman noise is observed to increase the $0 \mathrm{~dB}$ NF around $\lambda_{p}-\lambda_{s}=0 \mathrm{~nm}$ with $\sim 1.3 \mathrm{~dB}$. Up to $\left|\lambda_{p}-\lambda_{s}\right|=20 \mathrm{~nm}$ a NF less than $2 \mathrm{~dB}$ is achievable.

Figure 1(b) shows the NF vs. position of a PI amplifier for two cases with Raman scattering and one without. After $z \approx 500 \mathrm{~m}$ the pump starts to deplete and the NFs decrease to approx. $-40 \mathrm{~dB}$. After the turning point $z \approx 680 \mathrm{~m}$, the signal starts giving energy back to the pump and the NFs increase rapidly for all cases. The lower-wavelength curve increases to large positive NF values, while the higher-wavelength curve returns to large negative values without reaching $0 \mathrm{~dB}$. By neglecting Raman scattering at lengths longer than the turning point results in a significant error. The constellation diagrams in the insets explain the drastic behaviour of the NF in the depleted pump regime; the black lines point toward the origo. 1) shows the circular shaped field ensembles in the linear gain regime, where the higherwavelength ensemble has experienced a larger Raman gain; in 2) the ensembles are squeezed in amplitude by the amplifier but broadened in phase, which causes the photon-number based SNR to increase; 3) shows that the higherwavelength ensemble is yet again squeezed in amplitude, while in 4) the lower-wavelength ensemble is squeezed in phase. The ensemble without Raman (not shown) is observed to return to the non-squeezed state of 1) at the output.

In conclusion, we show a method for describing Raman scattering and vacuum fluctuations in parametric amplifiers using classical equations. Using the approach, we show the effect of Raman scattering in the linear and depleted gain regime. In the latter, coherent input states are shown to be squeezed by the amplifier, thus improving the SNR.

Acknowledgement The Danish Research Council for Technology and Production Sciences (project 09-066562) is acknowledged for financial support.

\section{References}

1. Tong et al., "Ultralow noise, broadband phase-sensitive optical amplifiers, and their applications," IEEE Journal of Selected Topics in Quantum Electronics 18, 1016 - 1032 (2012).

2. McKinstrie et al., "Quantum noise properties of parametric processes," Optics Express 13, 4986 - 5012 (2005).

3. Voss et al., "Raman-effect induced noise limits on $\chi^{(3)}$ parametric amplifiers and wavelength converters," Journal of Optics B: Quantum and Semiclassical Optics 6, 762 - 770 (2004).

4. Voss et al., "Raman-noise-induced quantum limits for $\chi^{(3)}$ nondegenerate phase-sensitive amplification and quadrature squeezing," Journal of the Optical Society of America B: Optical Physics 23, 598 - 609 (2006).

5. Hsieh et al., "Combined effect of Raman and parametric gain on single-pump parametric amplifiers," Optics Express 15, $8104-8114$ (2007).

6. Hansryd et al., "Fiber-Based Optical Parametric Amplifiers and Their Applications," IEEE Journal of Selected Topics in Quantum Electronics 8, 506 - 520 (2002).

7. Kidorf et al., "Pump interactions in a 100-nm bandwidth Raman amplifier," Photonics Technology Letters 11, $530-532$ (1999). 